\title{
Liaison Psychiatry During Challenging Times
}

\author{
Ashika Bains $^{1}$ (ID \\ Received: 11 May 2020 / Accepted: 9 February 2021 / Published online: 2 March 2021 \\ (C) Academic Psychiatry 2021
}

Michigan was one of the harder hit states of the COVID-19 crisis. By early April, Detroit represented the largest caseload of viral infection outside of the East Coast. Amid worry over shortages of resources and personal protection equipment, healthcare workers struggled to rise to the challenge. The emerging question for us became the role of psychiatry during a pandemic: What could we do to help?

The literature on mental health during the COVID-19 pandemic discussed anxiety, depression, and traumatization for frontline workers [1]. My co-residents and I attempted to implement hotlines for access to mental health services for healthcare workers; however, despite attempts at reducing barriers to access, it became evident that there was a lag in engagement. Individuals were only reaching out when their struggle became unbearable. We wanted an intervention that could assist prior to burnout and despair.

With this in mind, three co-residents and I served in a liaison role to the intensive care unit teams to provide grief processing to the families of COVID-positive critically ill patients. Our training as psychiatrists was useful in distress management for families which eased emotional burden for our intensive care colleagues during a pandemic. Additionally, we were available to provide support for healthcare staff as needed. We rounded with the teams and took on liaison work between the physicians and families for certain cases (complex family dynamics, young patients, and end of life care). Faculty supervision was done by phone twice weekly; these meetings allowed us to process our own reactions to difficult cases and gain guidance on addressing the ego defenses we encountered in patient's families.

The crisis had overloaded the hospital with sick and dying patients, and ICU staff barely had time to process one death before having to move onto another. The constant stress

Ashika Bains

abains@med.wayne.edu

1 Wayne State University, Detroit, Michigan, USA resulted in feelings of loneliness, despair, burnout, and intense anxiety. Interventions we provided to assist included postround mini-lectures on reactions during traumatic events to normalize and validate emotions, hosting non-invasive impromptu group ventilation sessions in the work room, holding de-briefs after stressful/traumatic patient deaths or codes, and providing education on defense mechanisms that the staff may encounter in themselves or others. For instance, during a facilitated group discussion, physicians described losing themselves in their work, being too tired to do things they once enjoyed; some confessed having any emotions during a crisis made them feel weaker than their colleagues. During the conversation, it became clear that everyone was struggling with similar concerns which helped combat feelings of isolation and provided an in-road to discussions of self-care. Another illustration of a successful intervention occurred during a discussion about a family with a provider who voiced frustrations stating the family was "too emotional." The provider then spontaneously began talking about himself, relaying that he is usually someone who tears up at touching moments in movies, but lately has felt nothing. He wondered if he had lost his emotions entirely. Providing psychoeducation that numbness may be a coping mechanism and likely temporary helped alleviate his percolating anxiety. These were some of the ways, by being physically present at the right moments, we were able to share our knowledge and assist our colleagues.

The most gratifying work we did was with the families. Due to the quarantine, hospitals were no longer allowing visitors resulting in anxiety and apprehension. Families described waiting by the phone for a call for an update on medical status while simultaneously dreading a call with bad news. Being unable to visit allowed family members to hold onto the image of their relative as not sick which delayed the potential for anticipatory grief and perpetuated defenses like denial. The interventions we provided were gentle reminders of illness with daily medical updates, assisting in the start of the narrative with questions like "how did she get sick?" to process shock, hosting tele-health videos on secure platforms, supportive counseling over the phone, recognizing defense 
mechanisms and responding appropriately, and mediating family meetings with the ICU teams for goals of care. In one instance, the sister of a patient repeatedly asked to be able to talk to the patient over the phone despite multiple physicians describing to her that the patient was intubated and sedated. My medical colleagues were frustrated and worried that the surrogate decision-maker for our patient was not comprehending the situation. When I spoke to her, it became apparent she was in denial about the severity of the patient's illness and challenging that defense only resulted in further denial. I discussed the utility of the coping mechanism with ICU team and became the intermediary for discussions. In speaking with the sister, I encouraged the start of the narrative by asking her questions about the patient; she described the patient's personality and medical history eventually conceding to how angry she was that the patient continued to take the bus and caught the virus despite warnings from multiple family members. We discussed her conflicting emotions. This supportive measure coupled with secure video conferencing allowed her to see the patient on the ventilator and facilitated reality orientation. She became less defensive with the doctors and participated in a meeting on goals of care.

Working alongside our internal medicine colleagues was an intense and amazing learning experience. Intensive care workers continue to astonish and inspire me with their courage and diligence. The teams were welcoming and appreciated my input and interpretations of cases and behaviors, which led me to realize the importance of liaison work of psychiatry. I gained an appreciation for bridging the gap between the specialties of medicine and psychiatry and provided mental health support for my colleagues during a crisis. By physically being present in that environment, we were able to shoulder some of the burden for our community. In Detroit, we hope for better things, and it will rise from the ashes.

\section{Declarations}

Disclosures The author states that there is no conflict of interest.

\section{References}

1. Rajkumar RP. COVID-19 and mental health: A review of the existing literature. Asian J Psych. 2020;52:102066.

Publisher's Note Springer Nature remains neutral with regard to jurisdictional claims in published maps and institutional affiliations. 